\title{
Inflation from Quantum Geometry
}

\author{
Martin Bojowald \\ Center for Gravitational Physics and Geometry, The Pennsylvania State University, \\ 104 Davey Lab, University Park, PA 16802, USA
}

\begin{abstract}
Quantum geometry predicts that a universe evolves through an inflationary phase at small volume before exiting gracefully into a standard Friedmann phase. This does not require the introduction of additional matter fields with ad hoc potentials; rather, it occurs because of a quantum gravity modification of the kinetic part of ordinary matter Hamiltonians. An application of the same mechanism can explain why the present-day cosmological acceleration is so tiny.
\end{abstract}

Inflation (an accelerated phase of the universe, i.e. $\ddot{a}>0$ for the scale factor $a$ ) has been proposed as an elegant solution of severe problems of the cosmological standard model [1]. However, it suffers from the lack of a natural explanation from fundamental physics. Usually, a scalar field, the inflaton, is proposed which requires a special potential and initial conditions for sufficient inflation. The inflationary phase is usually deemed to happen after the Planck epoch of the universe, but it has been speculated that there is a quantum gravity origin.

The first such realization employed effective higher curvature terms in the gravitational action [2] which leads to deSitter-like solutions, or inflation, even without introducing a cosmological constant explicitly. However, in this perturbative higher derivative theory not all solutions are reliable and non-analytic ones in the perturbation parameter (the Planck length) have to be excluded [3]. In particular, this has to be done for the deSitterlike solutions since their effective cosmological constant is given by an inverse power of the Planck length.

A more recent idea [4] is to use modified dispersion relations, which are expected to appear in most quantum gravity theories, for an explanation of inflation. In fact, this is possible provided that the dispersion relation has a special form with the momentum decreasing to zero for large energies. Such dispersion relations have been proposed, but not yet derived from any theory of quantum gravity. Moreover, in current derivations of dispersion relations one obtains a power series in the energy which is inconclusive concerning the high energy behavior.

A lesson is that a non-perturbative approach would produce the most reliable answer as to whether or not inflation can be derived from quantum gravity. Currently, the most direct non-perturbative realization of a quantum theory of gravity is provided by quantum geometry (also called loop quantum gravity, [5]), which is a canonical quantization of general relativity in terms of Ashtekar's variables. In recent years, techniques to study its cosmological sector have been developed [8] which will be used here. We will see that quantum geometry can naturally lead to inflation at small volume with a graceful exit.

Loop quantum cosmology. By studying isotropic states of quantum geometry one obtains isotropic loop quantum cosmology which reproduces the standard
Wheeler-DeWitt approach at large volume [9], but leads to significant deviations at small volume caused by the discreteness of geometry. Notable consequences are the absence of cosmological singularities 10 and the prediction of 'dynamical initial conditions' for the wave function of a universe [11]. These already offer solutions to some of the pressing cosmological problems; here we ask what this implies for the occurrence of inflation.

In what follows we recall the basic formulae necessary for this investigation. Basic classical variables are the connection component $c$ and the isotropic triad component $p$. Because of the two possible orientations of the triad, $p$ can also take negative values, but later we will only need positive ones. It is related to the scale factor $a$ by $|p|=a^{2}$. For spatially flat models, which will only be used here, $c$ is proportional to the extrinsic curvature $k=\frac{1}{2} \dot{a}$. The variables $(k, p)$ form a canonical pair with $\{k, p\}=\frac{1}{3} \kappa, \kappa=8 \pi G$ being the gravitational constant. They appear in the gravitational part of the Hamiltonian constraint

$$
\mathcal{H}=-6 \kappa^{-1} k^{2} \sqrt{p}+\mathcal{H}_{\phi}
$$

where $\mathcal{H}_{\phi}$ is the matter Hamiltonian for a field $\phi$ (this represents a standard matter field and need not be an inflaton) which for simplicity will be taken to be of the standard form $\mathcal{H}_{\phi}=\frac{1}{2} a^{-3} p_{\phi}^{2}+a^{3} V(\phi)$. Here, $V(\phi)$ is an arbitrary potential and $p_{\phi}=a^{3} \dot{\phi}$ the momentum canonically conjugate to $\phi$. The Hamiltonian constraint $\mathcal{H}$ generates the dynamics of the theory and gives the Friedmann equation after using $k=\frac{1}{2} \dot{a}$.

A standard Wheeler-DeWitt quantization would proceed by using wave functions $\psi(p, \phi)$. Consequently, $\hat{p}$ has a continuous spectrum containing zero and, after choosing the scale factor as internal time, the Hamiltonian constraint turns into a second order differential time evolution equation, the Wheeler-DeWitt equation

$$
-\frac{2}{3} l_{\mathrm{P}}^{4} \frac{\partial^{2}}{\partial p^{2}}(\sqrt{p} \psi) \equiv-\frac{1}{6} l_{\mathrm{P}}^{4} a^{-1} \frac{\partial}{\partial a} a^{-1} \frac{\partial}{\partial a}(a \psi)=\kappa \hat{\mathcal{H}}_{\phi} \psi .
$$

The matter Hamiltonian $\hat{\mathcal{H}}_{\phi}$ is an unbounded operator owing to the kinetic term containing an inverse power of the scale factor. For the matter variables we can choose a standard quantization which promotes $\phi$ to a multiplication operator and $p_{\phi}$ to the derivative operator $-i \hbar \partial / \partial \phi$. 
We will use the same matter field quantization for loop quantum cosmology. Concerning the geometrical degrees of freedom, however, the situation is very different. Most notably, the spectrum of geometric operators like $\hat{a}$ is discrete, which is directly inherited from the full theory of quantum geometry. This implies that, in a metric representation, the wave function $s_{n}(\phi)$ replacing $\psi(p, \phi)$ lives on a discrete space labeled by an integer $n$ representing the geometry. (Again, the sign of $n$ is the orientation, but later only positive $n$ will be needed.) The integer $n$ also appears in the discrete eigenvalues of $\hat{p}$ which have the form

$$
p_{n}=\frac{1}{6} \gamma l_{\mathrm{P}}^{2} n
$$

where a new parameter $\gamma$, the Barbero-Immirzi parameter, occurs. It is a positive real number which sets the scale for the discreteness of geometry and thus plays the role of a new dimensionless fundamental constant which controls the continuum limit $(\gamma \rightarrow 0$ and $n \rightarrow \infty)$ just as $\hbar$ controls the classical limit [9]. Its value $\gamma=\ln 2 / \pi \sqrt{3} \approx$ 0.13 has been determined by comparing the black hole entropy obtained from quantum geometry with the semiclassical result [12,13]. Also the volume operator $\hat{V}$ has a discrete spectrum with eigenvalues 14

$$
V_{\frac{1}{2}(n-1)}=\left(\frac{1}{6} \gamma l_{\mathrm{P}}^{2}\right)^{\frac{3}{2}} \sqrt{(n-1) n(n+1)} .
$$

Both operators $\hat{p}$ and $\hat{V}$ have eigenvalue zero for $n=0$. Therefore, the obvious definition of their inverse fails and we have to face the problem of how to quantize the kinetic part of the matter Hamiltonian which contains the inverse volume.

Inverse volume operators. It turns out 15] that there is a well-defined, finite quantization of inverse powers of the scale factor in loop quantum cosmology, which makes use of a general technique of full quantum geometry [16,17]. This can be interpreted as providing a natural curvature cut-off and plays a crucial role in the proof of absence of cosmological singularities [10]. Here we illustrate this technique in the isotropic context: Using the symplectic structure it is easy to see that the scale factor can be written as $a=2 \kappa^{-1}\{k, V\}$. This implies

$$
a^{-1}=a V^{-\frac{2}{3}}=2 \kappa^{-1} V^{-\frac{2}{3}}\{k, V\}=6 \kappa^{-1}\left\{k, V^{\frac{1}{3}}\right\}
$$

where the negative power of the volume has been absorbed into the Poisson bracket. This reformulation allows a quantization by expressing $k$ in terms of a holonomy of the Ashtekar connection (which is a basic operator in quantum geometry), using the volume operator and turning the Poisson bracket into a commutator. However, since the expression for $a^{-1}$ is a rather complicated function of the basic variables, the final quantization contains quantization ambiguities of different types. Most of them only affect the lowest eigenvalues for small $n$ of the order one; but there is one, resulting from the use of arbitrary representations with label $j$ for the holonomy, which can also affect higher eigenvalues [18]. This quantization ambiguity provides the mechanism which will be exploited here to obtain inflation in an effective classical description; as with all quantization ambiguities, the final judgement about their value has to come from observations. We will employ a quantization $\hat{d}_{j}$ of the density $d=a^{-3}$ which has eigenvalues (see Fig. 畐)

$$
d_{j, n}=\left[12\left(j(j+1)(2 j+1) \gamma l_{\mathrm{P}}^{2}\right)^{-1} \sum_{k=-j}^{j} k \sqrt{V_{\frac{1}{2}(|n+2 k|-1)}}\right]^{6}
$$

labeled by the ambiguity parameter $j$, a half-integer (we use an operator $\hat{d}_{j}$ which is the sixth power of the operator $\hat{s}_{j}$ of [18]).

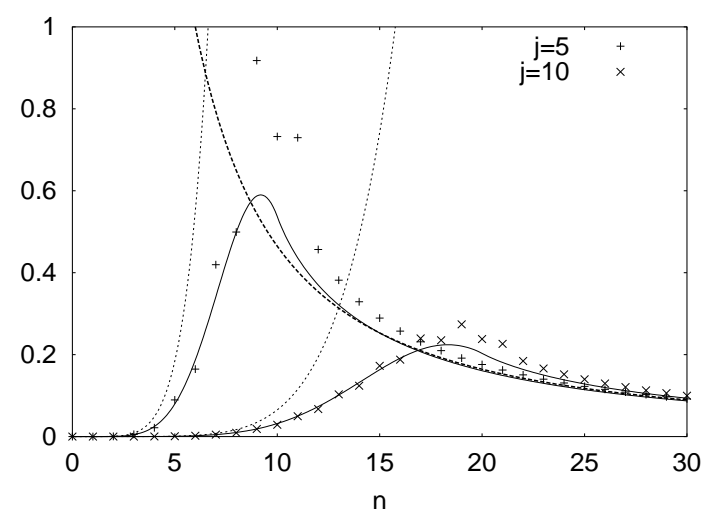

FIG. 1. Eigenvalues $d_{j, n}$ of the density operator $\hat{d}_{j}$ (in units of $\left.\left(\gamma l_{\mathrm{P}}^{2}\right)^{-\frac{3}{2}}\right)$ for two different $j$, compared to the approximation $p(n / 2 j)^{6} V_{\frac{1}{2}(n-1)}^{-1}$ (solid lines) and the classical expectation $V_{\frac{1}{2}(n-1)}^{-1}$ (thick dashed line). The dotted lines are the approximations (4) for small $n$.

An approximation, which gets better for large $j$, is obtained as $d_{j, n} \simeq V_{\frac{1}{2}(n-1)}^{-1} p(n / 2 j)^{6}$ where the function

$$
\begin{aligned}
p(q)= & \frac{8}{77} q^{\frac{1}{4}}\left(7\left((q+1)^{\frac{11}{4}}-|q-1|^{\frac{11}{4}}\right)\right. \\
& \left.-11 q\left((q+1)^{\frac{7}{4}}-\operatorname{sgn}(q-1)|q-1|^{\frac{7}{4}}\right)\right)
\end{aligned}
$$

has been derived in [18]. This formula has the asymptotic expansions

$$
d_{j, n} \simeq \frac{12^{6}}{7^{6}} V_{\frac{1}{2}(n-1)}^{-1}(n / 2 j)^{\frac{15}{2}}
$$

for $n \ll 2 j$ and $d_{j, n} \simeq V_{\frac{1}{2}(n-1)}^{-1}$ for $n \gg 2 j$. The approximation (4) shows how the classical divergence of the inverse volume is truncated by the large power of $n$. In particular, the dependence on $n$ of the density $d_{j}$ is very different at small and large $n$ which, when used in the matter Hamiltonian, implies severe changes of the cosmological evolution near the classical singularity. If $j$ 
is larger than one, we can study this evolution by an effective matter Hamiltonian which incorporates these different behaviors. To this end, we write the density as a function of $a$ instead of $n$ using the relation $a^{2}=\frac{1}{6} \gamma l_{\mathrm{P}}^{2} n$ which follows from (2). This yields

$$
d_{j}(a)=a^{-3} p\left(3 a^{2} / \gamma l_{\mathrm{P}}^{2} j\right)^{6}
$$

with approximations

$$
d_{j}(a) \simeq \frac{12^{6}}{7^{6}}\left(\frac{1}{3} \gamma l_{\mathrm{P}}^{2} j\right)^{-\frac{15}{2}} a^{12}
$$

for $a^{2} \ll \frac{1}{3} \gamma l_{\mathrm{P}}^{2} j$ and $d_{j}(a) \simeq a^{-3}$ for $a^{2} \gg \frac{1}{3} \gamma l_{\mathrm{P}}^{2} j$. The approximation for small $a$ disappears in the continuum limit $\gamma \rightarrow 0$, in which case we only obtain the classical behavior $a^{-3}$.

Effective Friedmann equation. Since the spectra of geometric operators in loop quantum cosmology are discrete, the evolution equation in an internal time variable will also be discrete, i.e. a difference rather than a differential equation [19]. Its main part is of the form [8]

$$
\begin{aligned}
& (\mathcal{D} s)_{n}(\phi):=\left(V_{\frac{1}{2}(n+4)}-V_{\frac{1}{2}(n+4)-1}\right) s_{n+4}(\phi) \\
& -2\left(V_{\frac{1}{2} n}-V_{\frac{1}{2} n-1}\right) s_{n}(\phi)+\left(V_{\frac{1}{2}(n-4)}-V_{\frac{1}{2}(n-4)-1}\right) s_{n-4}(\phi) \\
& =-\frac{1}{3} \gamma^{3} \kappa l_{\mathrm{P}}^{2} \hat{\mathcal{H}}_{\phi}(n) s_{n}(\phi)
\end{aligned}
$$

where $n$ (analogous to $p$ ) is the discrete internal time (negative $n$, which are not being considered here, correspond to time before the classical singularity [10]). If the wave function is not oscillating at small scales (from $n$ to $n+1$ ), the difference operator $\mathcal{D}$ can be approximated by a differential operator which turns out to be $3 \gamma^{-3} l_{\mathrm{P}}^{-2} \mathcal{D} \approx \frac{2}{3} l_{\mathrm{P}}^{4} \frac{\partial^{2}}{\partial p^{2}} \sqrt{p}$, i.e. the operator appearing in the standard Wheeler-DeWitt equation [9]. Noting that $\frac{1}{3} i l_{\mathrm{P}}^{2} \frac{\partial}{\partial p}$ quantizes $k=\frac{1}{2} \dot{a}$, the resulting evolution equation corresponds to the Friedmann equation $\frac{3}{2} \dot{a}^{2} a=\kappa \mathcal{H}_{\phi}(a)$. The matter Hamiltonian, however, does not have the Wheeler-DeWitt form for all $a$ because we were forced to quantize the inverse volume to the welldefined operator $\hat{d}_{j}$ ( $j$ can be regarded as a new quantum number characterizing the matter component). Therefore, instead of the density $a^{-3}$ we have the effective density $d_{j}(a)$ from (5) which is modified at small $a$. Our Friedmann equation for $H=\dot{a} / a$ then is

$$
H^{2}=\frac{2}{3} \kappa a^{-3} \mathcal{H}_{\phi}^{(j)}(a)=\frac{2}{3} \kappa\left(\frac{1}{2} a^{-3} d_{j}(a) p_{\phi}^{2}+V(\phi)\right)
$$

which will be studied in the remainder of this paper.

Super-inflation. To simplify the analysis we first assume that the matter field $\phi$ is a free massless field, i.e. $V(\phi)=0$. Then, $p_{\phi}$ is constant and at large volume we have the Friedmann phase $a(t) \propto\left(t-t_{0}\right)^{1 / 3}$. If $a^{2}$ is small compared to $\frac{1}{3} \gamma l_{\mathrm{P}}^{2} j$, however, the density behaves as in (6) which leads to super-inflationary expansion 20] $a(t) \propto\left(t_{0}-t\right)^{-\frac{2}{9}}=\left(t_{0}-t\right)^{\frac{2}{3(1+w)}}$ with an equation of state parameter $w=-4$. ( $p_{\phi}$ has to be non-zero, but even a quantum fluctuation would suffice to get the inflationary growth started.) There is a pole at $t=t_{0}$, but it is never reached because the small- $a$ approximation breaks down when $a$ becomes larger. In fact, the superinflationary phase automatically ends when the peak of $d_{j}(a)$ is reached, exhibiting a graceful exit into the Friedmann phase (Fig. 2). Note also that $w$ is not constant during inflation; in fact it varies smoothly between -4 for small $a$ and $w=1$ for large $a$. A non-zero potential does not change this behavior qualitatively; depending on its form, however, it could lead to a second phase of potential driven inflation. This would occur more generically than in the standard scenario because the first phase can drive the scalar field up the potential hill, even if $\phi$ starts at the value $\phi=0$.

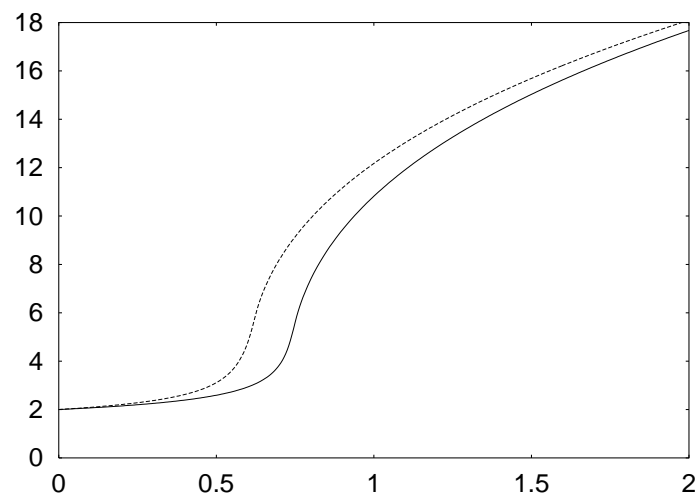

FIG. 2. Numerical solutions $a(t)$ (in units of $\sqrt{\gamma} l_{\mathrm{P}}$, with an arbitrary scale for $t$ ) of the effective Friedmann equation (8) for $V(\phi)=0$ (solid) and a small quadratic potential (dashed), both with $j=100$.

An inflationary expansion can also be seen from the wave function $s_{n}(\phi)$ (Fig. 3; see 21] for details). At small $n$ the amplitude of the oscillations and the oscillation length decrease toward larger $n$ (indicating acceleration). After this phase (for $n>2 j=400$ in the figure), the oscillation length increases (deceleration).

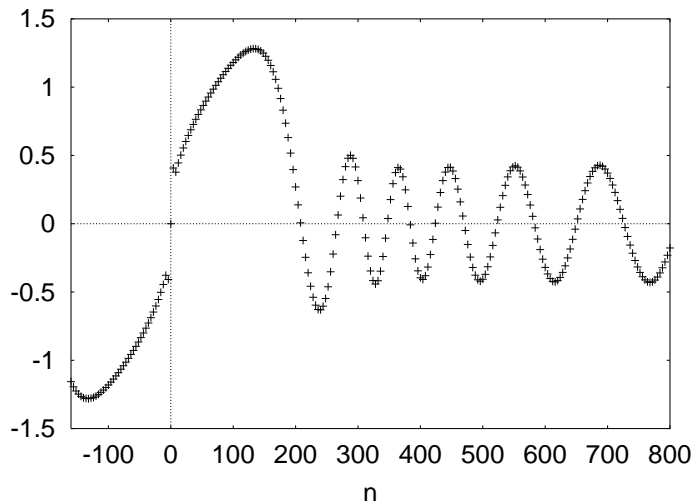

FIG. 3. An example for the discrete wave function $s_{n}(\phi)$ with a free massless scalar field and $j=200$. Negative $n$ represent time before the classical singularity.

The appearance of an inflationary phase is a direct consequence of the modified behavior of the density $d_{j}(a)$ for $a^{2}<\frac{1}{3} \gamma l_{\mathrm{P}}^{2} j$. This shows that such a phase disap- 
pears in the continuum limit $\gamma \rightarrow 0$ (which leads to Wheeler-DeWitt quantum cosmology) or in the classical limit $l_{\mathrm{P}} \rightarrow 0$. It is only possible if we incorporate the discreteness which requires $\gamma>0$, and $l_{\mathrm{P}}>0$.

The coefficient $w$ which determines the rate of superinflationary growth is not unique and depends on the quantization choices. Instead of $\hat{d}_{j}$ one can, e.g., use an operator $\hat{d}_{m, j}$ with eigenvalues

$d_{m, j, n}=\left[12\left(j(j+1)(2 j+1) \gamma l_{\mathrm{P}}^{2}\right)^{-1} \sum_{k=-j}^{j} k V_{\frac{1}{2}(|n+2 k|-1)}^{m}\right]^{\frac{3}{2-3 m}}$

with $0<m<\frac{2}{3}$ whose eigenvalues at large $n$ drop off as $n^{-\frac{3}{2}}$. At small $a$ the behavior is $d_{m, j}(a) \propto a^{l}$ where the power $l=\frac{6}{2-3 m}$ is bounded from below by $l>3$. This leads always to super-inflationary growth $a \propto\left(t_{0}-t\right)^{\frac{2}{3-l}}$ with coefficient $w=-l / 3$ but can get arbitrarily close to standard inflation $(w=-1)$. In fact one can also obtain phases of standard or power-law inflation [22], though in a less natural way (e.g. by using $\hat{d}_{1} \hat{d}_{j, 1 / 3}^{2 / 3} \hat{V}^{2 / 3}$ which grows as $a^{3}$ for $\left.\gamma l_{\mathrm{P}}^{2} \ll a^{2} \ll \gamma l_{\mathrm{P}}^{2} j\right)$.

Remarks. One may wonder how a quantum gravity effect can influence the universe expansion at large scales without a violation of the classical limit. This is possible because the limit $l_{\mathrm{P}} \rightarrow 0$ must include $n \rightarrow \infty$ in order to keep $a^{2}=\frac{1}{3} \gamma l_{\mathrm{P}}^{2} n$ non-zero. For $n \rightarrow \infty$ we are always in a Friedmann phase, no matter how large $j$ is. However, $l_{\mathrm{P}}$ is non-zero and the classical limit must not be performed completely. This leaves open the possibility of quantum gravity effects at large volume.

Choosing very large values for the parameter $j$ leads to matter Hamiltonians with kinetic terms which even today are still growing with $a$ and so exhibit a superinflationary phase at the present time (this matter component would behave as "phantom matter" with $w<-1$ [23]). While this involves huge $j$ and so is less natural, it can explain why today's cosmological constant is so small thanks to a suppression by an inverse power of $j$ : If we assume the potential term to be negligible, i.e. $V(\phi) \approx 0$ and thus $p_{\phi} \approx$ const., the Friedmann equation (8) implies

$$
H^{2} \propto\left(a / \sqrt{j} l_{\mathrm{P}}\right)^{9}\left(\sqrt{j} l_{\mathrm{P}}\right)^{-6} p_{\phi}^{2}
$$

when the small- $a$ approximation is valid. Here, the first factor is smaller than one thanks to $a^{2}<\frac{1}{3} \gamma l_{\mathrm{P}}^{2} j$ in the accelerated phase such that $H^{2}<\left(\sqrt{j} l_{\mathrm{P}}\right)^{-6} p_{\phi}^{2}$ which is well below the Planck mass for large $j$.

Conclusions. In this paper we presented what we believe is the first direct derivation of inflation from a candidate for a quantum theory of gravity. The arguments have been given in the context of isotropic models, but we only used techniques and generic properties which apply in full quantum geometry. Therefore, inflation with a graceful exit into a standard Friedmann phase can be regarded as a natural prediction of quantum geometry.
In fact, the inverse volume increases in a neighborhood of $a=0$ which leads to an accelerated expansion after evolving through $a=0$ (which is not a singularity in loop quantum cosmology $[10$ ). The modified behavior of the inverse volume is always present for small values of $a$ below the Planck scale (even for the minimal $j=\frac{1}{2}$ ). However, in this regime the classical space-time picture breaks down and the evolution cannot be described simply by a scale factor. This region has to be better understood before one can find a reliable estimate of the scale factor at the start of inflation. While it is clear that inflation ends when the scale factor reaches a value $a^{2} \approx \frac{1}{3} \gamma l_{\mathrm{P}}^{2} j$, its beginning lies in the Planck regime and can in principle be arbitrarily close to zero. Its value determines the number of $e$-foldings of the inflationary phase. The question of how inflation can be described in the quantum regime and its implications for fluctuations deserve further study.

The same effect can explain the present-day cosmological acceleration. While this appears less natural, it implies that if the mechanism proposed here is responsible, today's cosmological acceleration has to be small.

Acknowledgements. The author is grateful to A. Ashtekar for a critical reading of the manuscript. This work was supported in part by NSF grant PHY00-90091 and the Eberly research funds of Penn State.

E-mail address: bojowald@gravity.psu.edu

[1] A. H. Guth, Phys. Rev. D 23, 347 (1981).

[2] A. A. Starobinsky, Phys. Lett. B 91, 99 (1980).

[3] J. Z. Simon, Phys. Rev. D 41, 3720 (1990).

[4] S. Alexander, R. Brandenberger, and J. Magueijo, hepth/0108190

[5] A. Ashtekar, Lectures on non-perturbative canonical gravity (World Scientific, Singapore, 1991).

[6] C. Rovelli, Liv. Rev. Rel. 1, 1 (1998).

[7] T. Thiemann, gr-qc/0110034.

[8] M. Bojowald, Class. Quantum Grav. 19, 2717 (2002).

[9] M. Bojowald, Class. Quantum Grav. 18, L109 (2001).

[10] M. Bojowald, Phys. Rev. Lett. 86, 5227 (2001).

[11] M. Bojowald, Phys. Rev. Lett. 87, 121301 (2001).

[12] A. Ashtekar, J. C. Baez, A. Corichi, and K. Krasnov, Phys. Rev. Lett. 80, 904 (1998).

[13] A. Ashtekar, J. C. Baez, and K. Krasnov, Adv. Theor. Math. Phys. 4, 1 (2001).

[14] M. Bojowald, Class. Quantum Grav. 17, 1509 (2000).

[15] M. Bojowald, Phys. Rev. D 64, 084018 (2001).

[16] T. Thiemann, Class. Quantum Grav. 15, 839 (1998).

[17] T. Thiemann, Class. Quantum Grav. 15, 1281 (1998).

[18] M. Bojowald, gr-qc/0206053.

[19] M. Bojowald, Class. Quantum Grav. 18, 1071 (2001).

[20] F. Lucchin and S. Matarrese, Phys. Lett. B 164, 282 (1985).

[21] M. Bojowald and F. Hinterleitner, Isotropic Loop Quantum Cosmology with Matter, in preparation.

[22] F. Lucchin and S. Matarrese, Phys. Rev. D 32, 1316 (1985).

[23] R. R. Caldwell, astro-ph/9908168. 